\title{
An Electrochemical Study of Bulk and Nanowire Morphologies of Electrodeposited Polypyrrole
}

\author{
Karen Herdman* and Carmel B. Breslin* \\ *Electrochemistry and Materials Chemistry Research Group, Department of Chemistry, \\ National University of Ireland, Maynooth, County Kildare, Ireland.
}

Pyrrole was electrochemically polymerised onto gold electrodes at a fixed potential of $0.80 \mathrm{~V}$ vs. SCE to generate polypyrrole (PPy). Depending on the $\mathrm{pH}$ of the solution, PPy, was deposited in either a bulk or nanowire conformation. Electrochemical impedance spectroscopy, EIS, experiments were subsequently carried out on a bare gold electrode, PPy nanowires and bulk PPy of two different film surface areas. The impedance data were analysed using equivalent circuits giving information on the electrochemical properties of the polymers. The influence of different electrolytes on the impedance was also examined.

\section{Introduction}

Polypyrrole is a conducting polymer that has a wide range of applications including energy storage systems, e.g., supercapacitors and rechargeable batteries (1). Conducting polymers have conjugated systems in which electrons are delocalized over the conjugated system allowing charge to spread over the polymer back bone (2) resulting in electrical conductivity. The properties of polypyrrole depend on its morphology which is determined by the synthesis conditions (3). The simple electrochemical preparation results in a polymer of porous material with a large surface area and good conductivity (4). Dopant ions are used to restore neutrality during polymerisation and the nature of the dopant used affects the conductivity of the polymer (5).

Nanowire morphologies of PPy have many advantages over bulk polymers. These include increased electrical, structural and electrochemical properties (6). Nano sized materials have the added benefit of possessing larger surface areas which lead to an increased double layer capacitance (7). This is due to the reduced distance within the nanowire PPy over which the electrolyte must transport ions (8). They also have a lower diffusion resistance than a bulk PPy modified electrode (9).

Electrochemical impedance spectroscopy is a reliable powerful technique used to investigate the electrochemical characteristics of an electrochemical system, including double layer capacitance, diffusion, solution resistance and the determination of the rate of charge transfer processes (10). The computerised results are fitted to equivalent circuits that model the electrical responses of the system over a range of frequencies. The individual elements of the circuit model represent the electrochemical parameters of the polymer/electrode system (11).

The aim of this paper is to compare the properties of bulk and nanowire conformations of PPy using similar electropolymerisation conditions, and also bulk and nanowire 
conformations adapted to have similar electroactive surface areas. The electrochemical properties of the polymers were examined using cyclic voltammetry and electrochemical impedance spectroscopy.

\section{Experimental}

\section{$\underline{\text { Materials and Instruments }}$}

PPy was synthesised using a standard three-electrode electrochemical cell, consisting of a gold working electrode, a Pt wire auxiliary electrode and a saturated calomel reference electrode (SCE). All potentials quoted are with respect to SCE. The potentiostat used was a Solartron 1285 using Corrware software. Scanning electron microscopy (SEM) micrographs were obtained using a Hitachi S-3200-N with a tungsten filament electron source, using INCA-Act software ${ }^{\mathrm{TM}}$. All reagents used were of analytical grade and supplied by Sigma Aldrich.

\section{Modification of Electrode}

PPy nanowires were potentiostatically electrodeposited at $0.80 \mathrm{~V}$ vs. SCE from a $0.075 \mathrm{M}$ solution of pyrrole in an aqueous solution of $2.0 \mathrm{mM} \mathrm{LiClO}_{4}$ and $0.20 \mathrm{M}$ $\mathrm{Na}_{2} \mathrm{HPO}_{4}$. In order to obtain the bulk conformation of PPy the $\mathrm{pH}$ was adjusted using concentrated $\mathrm{HClO}_{4}$.

\section{Electrochemical Impedance Spectroscopy}

Electrochemical impedance spectroscopy, EIS, experiments were carried out in a three electrode electrochemical cell, using a Solartron Frequency Response Analyser (Model SI 1250) in conjunction with a Solartron potentiostat (Model SI 1287). The Frequency Response Analyser was controlled by ZPlot Version 2.1 for Windows ${ }^{\mathrm{TM}}$ and the resulting data were analysed with ZView Version 2.3 for Windows ${ }^{\mathrm{TM}}$. EIS studies of the PPy modified electrode were carried out in $0.20 \mathrm{M}$ solutions of $\mathrm{KCl}, \mathrm{LiClO}_{4}$ and $\mathrm{Na}_{2} \mathrm{HPO}_{4}$. All impedance measurements were carried out with an applied potential varying from $-0.50 \mathrm{~V}$ vs. SCE to $0.50 \mathrm{~V}$ vs. SCE, with a perturbation signal of $5 \mathrm{mV}$ and a frequency range of $65,000 \mathrm{~Hz}$ to $5 \mathrm{mHz}$. Each PPy modified electrode was examined immediately after electropolymerisation and was held at the given potential prior to the study for 30 min to ensure a steady state had been reached. The impedance data were fitted using equivalent circuits.

\section{Results and Discussion}

\section{$\underline{\text { Polymerisation }}$}

PPy nanowires were electropolymerised from a solution containing $0.075 \mathrm{M}$ pyrrole, $0.2 \mathrm{M} \mathrm{Na}_{2} \mathrm{HPO}_{4}$ and $2.0 \mathrm{mM} \mathrm{LiClO}_{4}$ for $300 \mathrm{~s}$ with an applied potential of $0.80 \mathrm{~V}$ vs. $\mathrm{SCE}$. The first bulk polymer was produced using similar conditions with the $\mathrm{pH}$ of the solution altered to 5.5. A second bulk polymer was formed so that its electroactive surface area was approximately the same as that of the nanowires. This was achieved using an electropolymerisation time of $11 \mathrm{~s}$. A low concentration of pyrrole was chosen in order to reduce the possibility of carbonyl defects in the conjugated back-bone of the 
polymer that have been reported to form from aqueous solutions (12). Figure 1 shows the current-time plots recorded during the formation of the nanowire and bulk polymers grown for $300 \mathrm{~s}$. For the bulk PPy there is an initial drop in the current on application of the applied potential, this is followed by a sharp rise in the current as the polypyrrole quickly nucleates and deposits onto the electrode surface. The current then reaches a near-steady state indicating efficient formation of the polymer. The data recorded for the nanowire polymer shows the same initial drop in current, but this continues until a steady state is reached. It is clear from a comparison of the two current-time transients in Figure 1 that the bulk polymer deposits at a high rate and the nanowire film is formed at a much slower rate. It is this slow growth that leads to the formation of nanowires and not bulk PPy.

Typical SEM micrographs of the nanowire and bulk polymers are shown in Figure 2. It can be seen that the morphologies of the bulk and nanowire polymers are very different. The bulk PPy has the typically cauliflower appearance (13) whereas the nanowires have formed with no visible agglomeration. They have an average diameter of $89.2 \mathrm{~nm}(\mathrm{n}=$ $50)$.

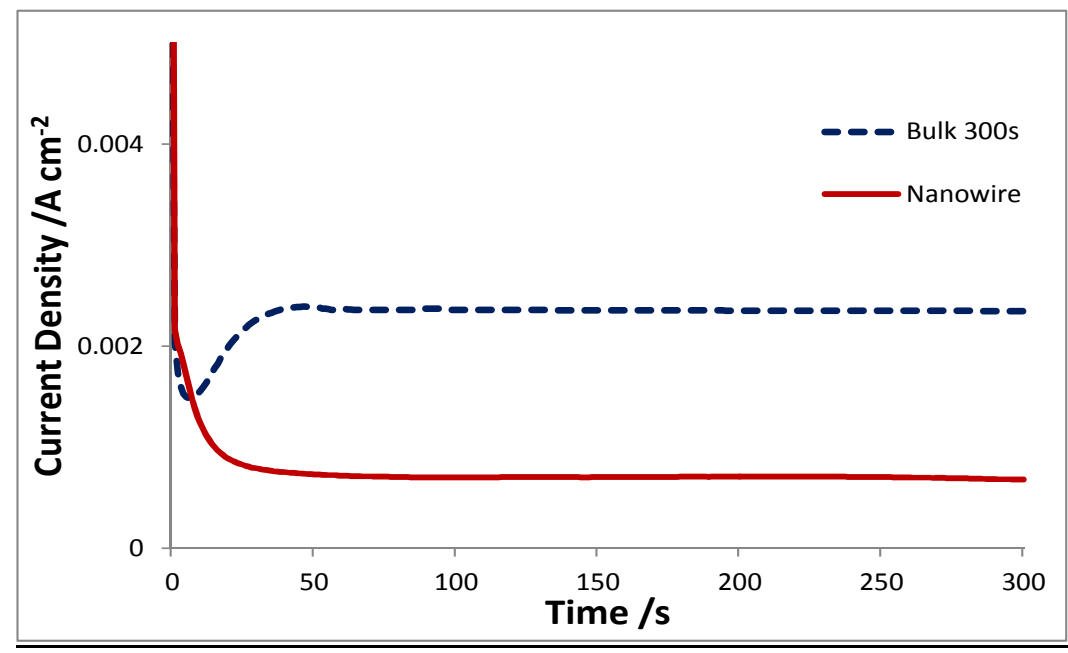

Figure 1. Current-time plots for bulk and nanowire PPy polymerised at $0.80 \mathrm{~V}$ vs. SCE for $300 \mathrm{~s}$.
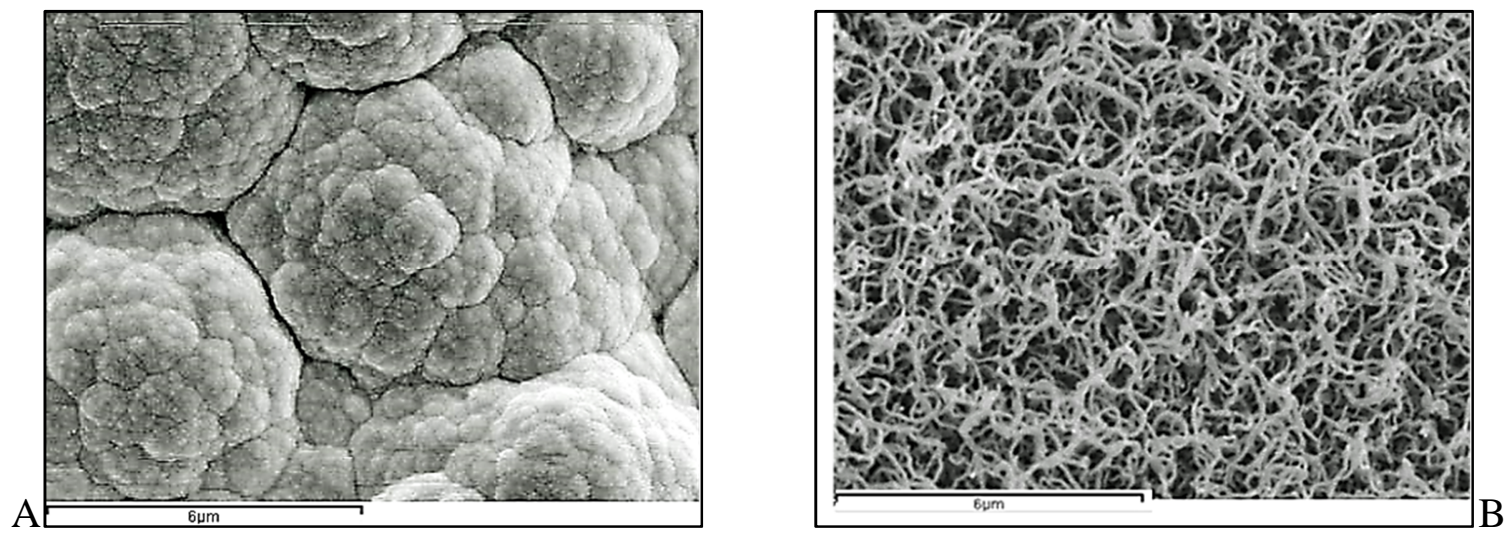

Figure 2. SEM micrographs of (A) bulk and (B) nanowire PPy. 


\section{Cyclic Voltammetry}

The redox activity of the bulk and nanowire modified electrodes was examined using cyclic voltammetry in $0.2 \mathrm{M} \mathrm{KCl}$. The results are shown in Figure 3, A and B. Figure 3A shows the cyclic voltammograms for bulk and nanowire PPy modified electrodes estimated to have the same electroactive surface area. Both polymers show quasireversibility. The bulk polymer has a slightly higher current. Figure 3B shows the voltammograms for the two polymers grown for $300 \mathrm{~s}$. The current is significantly higher for the bulk polymer showing that it possesses a higher capacitance. The voltammogram recorded for the nanowire morphology, shown in the inset of Figure 3B, shows clear oxidation and reduction peaks that exhibit a quasi-reversible system typical of conducting polymers (14). There are no visible peaks for the bulk PPy, as these have been masked by the high currents of the thick polymer.

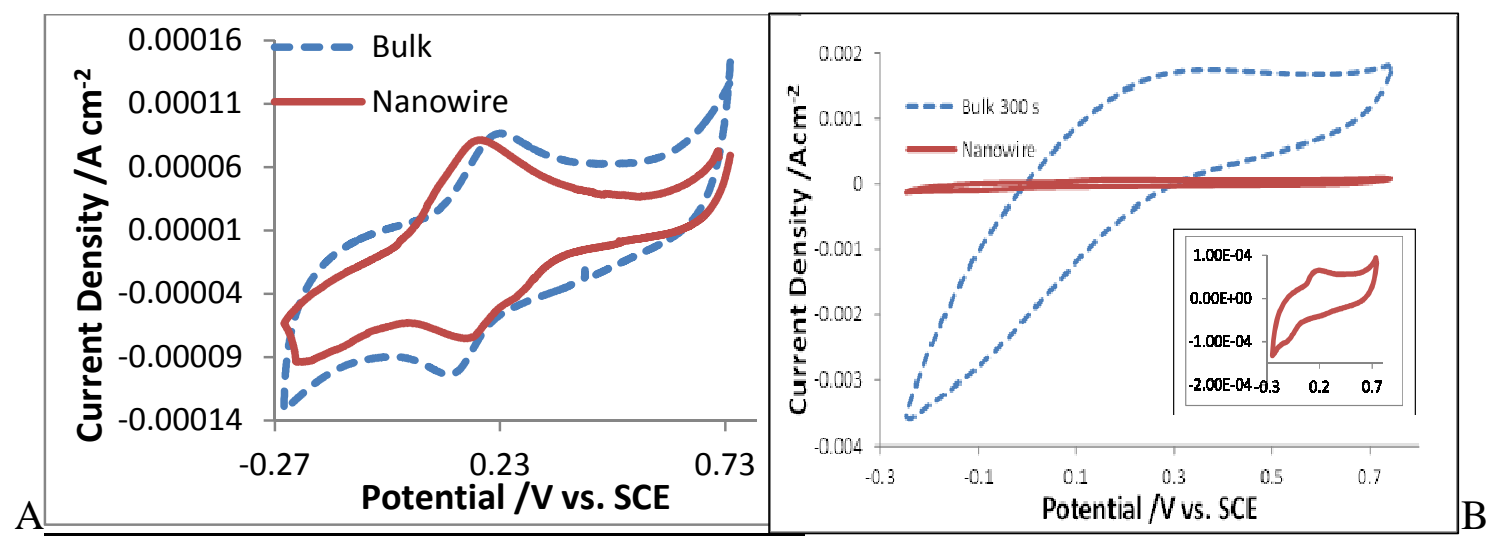

Figure 3. (A) Cyclic voltammograms of bulk (11 s) and nanowire (300 s) PPy grown at $0.80 \mathrm{~V}$ vs. SCE in $0.2 \mathrm{M} \mathrm{KCl}, \mathrm{v}=100 \mathrm{mV} / \mathrm{s}$. (B) Bulk and nanowire PPy both grown for $300 \mathrm{~s}$. The insert shows the nanowire CV magnified.

\section{$\underline{\text { Polymer Thickness }}$}

It was seen in Figure 3B that there were no visible oxidation and reduction peaks for the bulk polymer cycled in $0.2 \mathrm{M} \mathrm{KCl}$. This demonstrated that the capacitance was very much higher for the bulk PPy due to its much larger surface area. In order to grow a bulk polymer with approximately the same electroactive surface area as the nanowires, a calibration plot was prepared relating the polymer surface area with the charge consumed during electropolymerisation. Bulk polymers were grown for various times and the charge consumed during the electropolymerisation was recorded. Surface area estimations were made using the Randles-Sevcik (15) equation, Equation 1.

$$
\mathrm{Ip}=\left(2.69 \times 10^{5}\right) \mathrm{n}^{3 / 2} \mathrm{~A} \mathrm{C} \mathrm{D}^{1 / 2} v^{1 / 2}
$$

Here, Ip is the peak current (A), $\mathrm{n}$ is the number of electrons transferred, $\mathrm{A}$ is the surface area $\left(\mathrm{cm}^{2}\right), \mathrm{C}$ is the concentration of the redox species $(\mathrm{mol} \mathrm{cm}), \mathrm{D}$ is the diffusion coefficient $\left(\mathrm{cm}^{2} \mathrm{~s}^{-1}\right)$ and $v$ is the scan rate $\left(\mathrm{V} \mathrm{s}^{-1}\right)$. A plot of the charge density against the 
surface area resulted in a straight line plot, as shown in Figure 4, and the equation of the linear plot is given in Equation [2].

$$
y=0.3505 x+0.0946
$$

Using the Randles-Sevcik equation, Equation [1], the surface area of the nanowire modified electrode was estimated as $0.14 \mathrm{~cm}^{2}$. Similarly, using Equation [2], the bulk polymer grown for $300 \mathrm{~s}$ gave an estimated surface area of $0.74 \mathrm{~cm}^{2}$. In order to grow a bulk polymer with an estimated surface area the same as the nanowires the bulk polymer was grown for $11 \mathrm{~s}$. Accordingly, the electrochemical properties of the nanowire films were compared to a bulk PPy film formed for $11 \mathrm{~s}$.

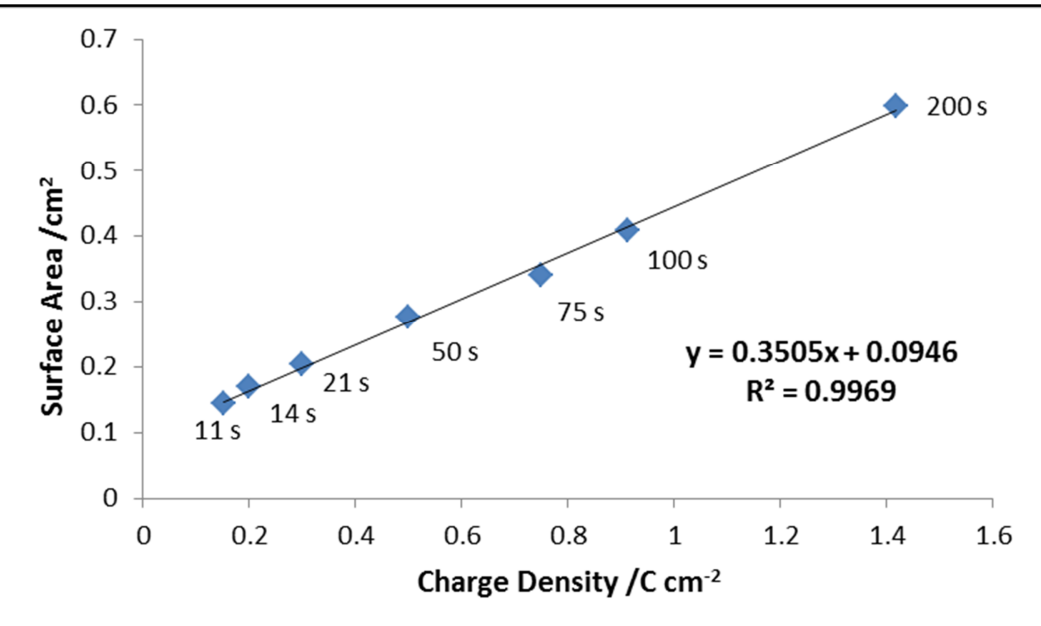

Figure 4. Calibration plot showing the relationship between the charge density consumed and the surface area of bulk PPy modified electrodes.

\section{Electrochemical Impedance Spectroscopy}

Electrochemical impedance spectroscopy experiments were carried out on a bare gold electrode, nanowire and two different bulk PPy modified electrodes in aqueous solutions of $0.2 \mathrm{M} \mathrm{KCl}, \mathrm{LiClO}_{4}$ and $\mathrm{Na}_{2} \mathrm{HPO}_{4}$. A potential range of $-0.50 \mathrm{~V}$ to $0.50 \mathrm{~V}$ vs. SCE was applied with a perturbation signal of $5 \mathrm{mV}$ and a frequency range of $65,000 \mathrm{~Hz}$ to $5 \mathrm{mHz}$ was used. The resulting impedance data for all bulk and nanowire conformations of the Ppy modified electrode were fitted to the same equivalent circuit shown in Figure 5, where Rs represents the solution resistance (11), CPE1 is a constant phase element that represents the double layer capacitance of the polymer/electrolyte interphase, R1 represents the polymer resistance and CPE2 is a constant phase element representing the capacitance of the polymer.

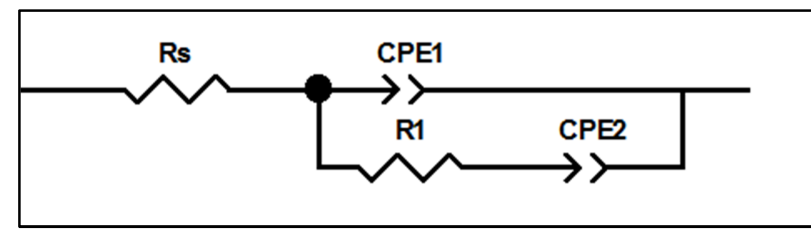

Figure 5. Equivalent circuit for bulk and nanowire Ppy modified electrodes. 
In Figure 6, Nyquist plots obtained for bare gold, bulk (formed for $11 \mathrm{~s}$ ) and nanowire PPy electrodes are compared. Once the bare electrode was modified with bulk or nanowire PPy, the impedance behaviour of the surface changed to semi circular plots in the high frequency range of the Nyquist plots, this is clearly shown in Figure 6 . The intercept of the semi circle with the $Z$ ' axis in the high frequency region indicates the solution resistance. The nanowire polymer has a region where the slope of the graph is approximately $45^{\circ}$, this is characteristic of ion diffusion in the porous structure of the polymer (10). The low frequency response for both polymers is almost a vertical line indicating that the polymers are almost purely capacitive. This is modelled by a constant phase element to allow for the dispersion of the line (16). Table I shows the double layer capacitance and the resistance and capacitance the polymer films and gold electrode. It is clear that these parameters have changed on modification of the electrode. There is an increase in the capacitance and a decrease of the resistance on modifying the gold electrode with the polymer films. The fact that the resistance decreased when modified with bulk PPy and shows a further decrease when modified with nanowires, indicates that the modification, especially with nanowires, has improved the electron transfer between the solution and the electrode (17).

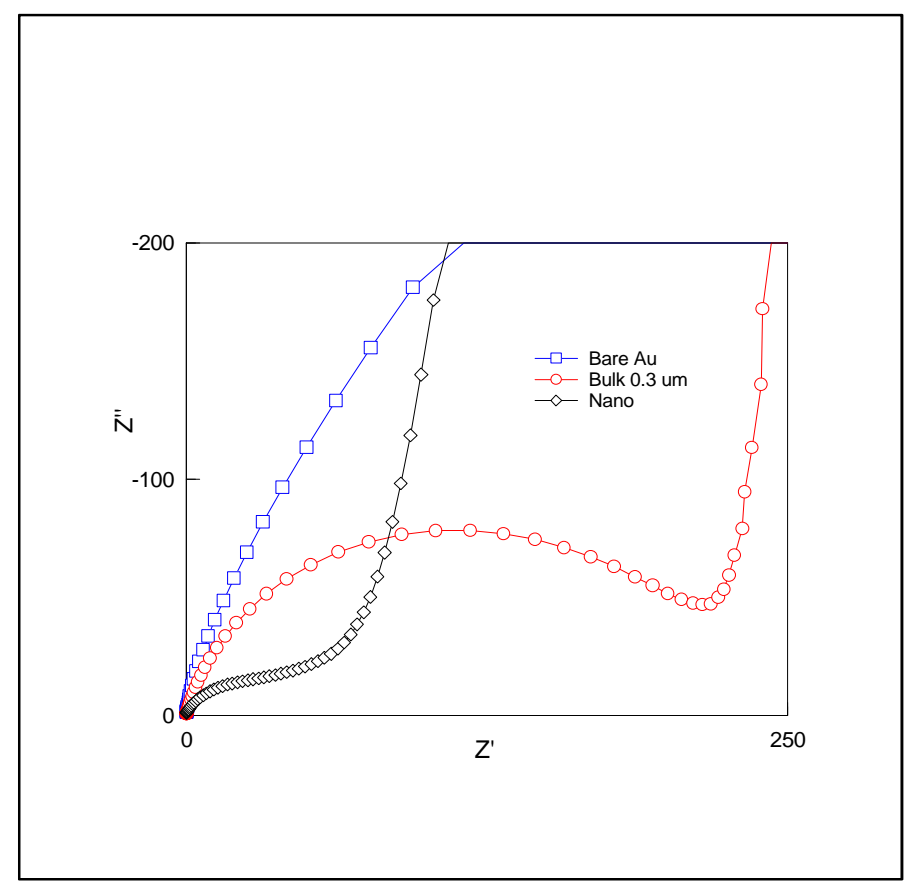

Figure 6. Nyquist plots obtained for bare gold, bulk (11 s) and nanowire PPy electrodes at O.C.P (0.06 V vs. SCE) in $0.2 \mathrm{M} \mathrm{LiClO}_{4}$.

TABLE I. Equivalent circuit values for bare gold, nanowire and bulk PPy modified electrodes at O.C.P. (0.06 V vs. SCE) in $0.2 \mathrm{M} \mathrm{LiClO}_{4}$.

\begin{tabular}{cccc}
\hline Surface & $\mathbf{C 1} / \mathbf{m F ~} \mathbf{~ m}^{-\mathbf{2}}$ & $\mathbf{R} 1 / \mathbf{\Omega} \mathbf{~ c m}^{\mathbf{2}}$ & $\mathbf{C 2} / \mathbf{m F ~ \mathbf { ~ m } ^ { - 2 }}$ \\
\hline Bulk PPy (11 s) & 0.99 & 228 & 6.9 \\
Nanowire PPy & 0.45 & 74 & 3.7 \\
Bare Au & $5.30 \times 10^{-3}$ & 1395 & 0.27 \\
\hline
\end{tabular}


EIS experiments were performed over a range of potentials, from $-0.50 \mathrm{~V}$ vs. SCE to $0.50 \mathrm{~V}$ vs. SCE, and at the open-circuit potential (O.C.P.) The O.C.P. after $30 \mathrm{~min}$ was $0.06 \mathrm{~V}$ vs. SCE. Figure 7 shows the response of a bulk (11 s) modified electrode in 0.2 $\mathrm{M} \mathrm{KCl}$ at a range of potentials. The impedance of the film is relatively independent of potential between $-0.1 \mathrm{~V}$ and $0.3 \mathrm{~V}$ vs. SCE. On decreasing the applied potential there is a large change in the slope of the low frequency constant phase element. This may indicate greater inhomogeneity in the ionic diffusion coefficient in the reduced state as the slope of a CPE is generally related to the porosity of the polymer (16).

Table II shows the impedance of a bulk PPy modified electrode recorded in $\mathrm{KCl}$, $\mathrm{LiClO}_{4}, \mathrm{Na}_{2} \mathrm{HPO}_{4}$ electrolyte solutions. The $\mathrm{pH}$ of these solutions ranged from 6.0 to 9.3. Similar double layer and polymer capacitances were obtained in the $\mathrm{KCl}(\mathrm{pH}=6.1)$ and $\mathrm{LiClO}_{4}(\mathrm{pH}=6.0)$ solutions. The alkaline $\mathrm{Na}_{2} \mathrm{HPO}_{4}(\mathrm{pH} 9.3)$ resulted in lower capacitance values, indicating that the polymer stores less charge in an alkaline solution. Similar capacitance values were observed on changing the $\mathrm{pH}$ of the $\mathrm{KCl}$ and $\mathrm{LiClO}_{4}$ solutions to that of $\mathrm{Na}_{2} \mathrm{HPO}_{4}$, indicating the significant role of the $\mathrm{pH}$ of the solution.

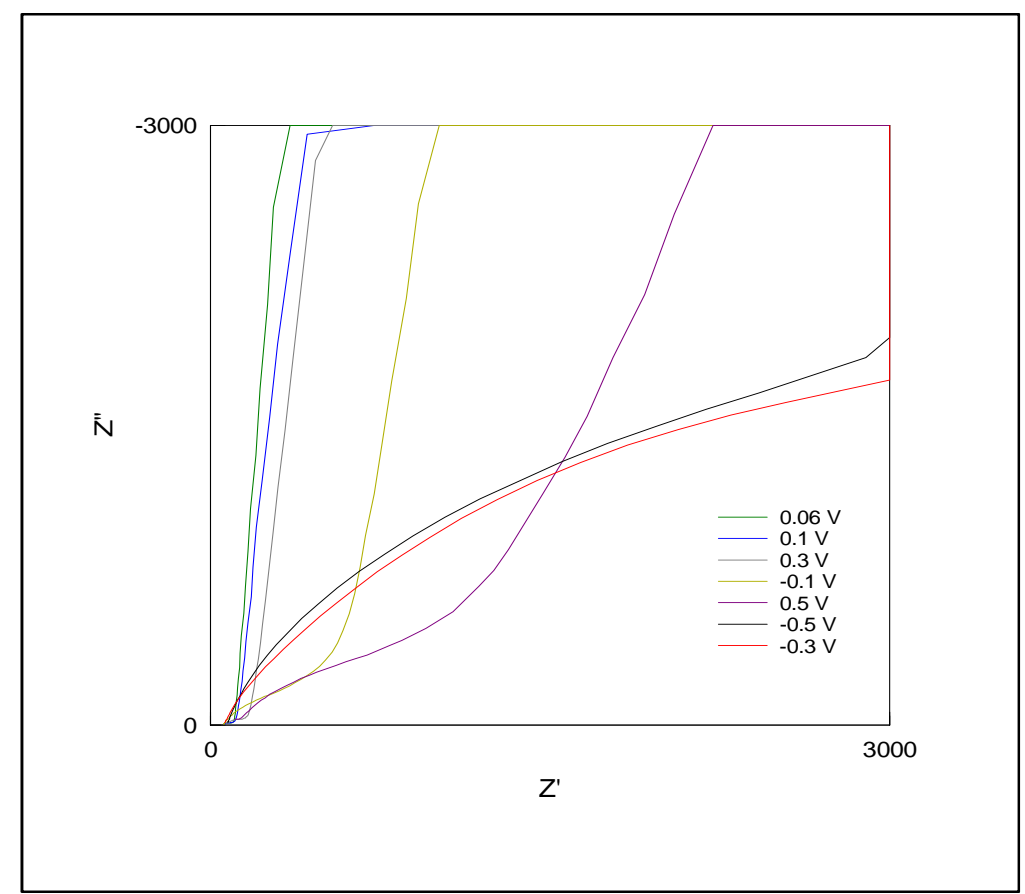

Figure 7. The effect of potential on a bulk PPy modified electrode in $0.2 \mathrm{M} \mathrm{KCl}$. The sequence of applied potential is read clockwise from left to right.

TABLE II. Effect of $\mathrm{pH}$ on resistance and capacitance values of bulk ( $300 \mathrm{~s})$ PPy at $0.10 \mathrm{~V}$ vs. SCE.

\begin{tabular}{ccccc}
\hline Electrolyte & $\mathbf{p H}$ & $\mathbf{C 1} / \mathbf{m F ~} \mathbf{~ m}^{-2}$ & $\mathbf{R 1} / \mathbf{\Omega} \mathbf{~ c m}^{2}$ & $\mathbf{C 2} / \mathbf{m F ~} \mathbf{~ m}^{-2}$ \\
\hline $\mathrm{KCl}$ & 6.1 & 5.6 & 3 & 67 \\
$\mathrm{LiClO}_{4}$ & 6.0 & 0.6 & 101 & 51 \\
$\mathrm{Na}_{2} \mathrm{HPO}_{4}$ & 9.3 & $9.3 \times 10^{-2}$ & 318 & 1.2 \\
$\mathrm{KCl}$ & 9.3 & $6.1 \times 10^{-2}$ & 66 & 0.3 \\
$\mathrm{LiClO}_{4}$ & 9.3 & $5.2 \times 10^{-2}$ & 77 & 5.4 \\
\hline
\end{tabular}




\section{Conclusion}

Pyrrole was electrodeposited onto gold electrodes in bulk and nanowire conformations. Two different sets of parameters were used to form the bulk polymers, the first using the same conditions but altering the $\mathrm{pH}$, and the second that resulted in a polymer of similar electroactive area to that of the nanowires. Cyclic voltammetry studies showed that the bulk polymer grown for $300 \mathrm{~s}$ had a much larger capacitance than the nanowires whereas the bulk polymer grown for $11 \mathrm{~s}$ had a similar capacitance. Electrochemical impedance spectroscopy studies showed how the electrochemical properties of the bare gold electrode had changed after modification with PPy. The effect of potential on the polymers was investigated, along with the effects of three different electrolytes on the PPy modified electrodes.

\section{Acknowledgments}

This work was funded by the Irish Research Council.

\section{References}

1. M. Shamsipur, H.K. Sayed and M. F. Mousavi, Biosensors and Bioelectronics, 24, 104, (2008).

2. J. Heinze, Electronically Conducting Polymers, 152, (1990).

3. J. Hegewald, L. Jakisch and J. Pionteck, Synthetic Metals, 159, 103, (2009).

4. V. Callegari and S. Demoustier-Champagne, Macromolecular Rapid Communications, 32, 25, (2011).

5. N. K. Guimard, N. Gomez and C. E. Schmidt, Progress in Polymer Science, 32, 876, (2007).

6. S. I. Cho and S. B. Lee, Accounts of Chemical Research, 41, 699, (2008).

7. C. Debiemme-Chouvy, Electrochemistry Communications, 11, 298, (2009).

8. Q. F. Wu K.X. He, H. Y. Mi and X. G. Zhang, Materials Chemistry and Physics, 101, 367, (2007).

9. X. Zhang, J. Wang, Z. Wang and S. Wang, Sensors, 5, 580, (2005).

10. M. Ates, Progress in Organic Coatings, 71, 1, (2011).

11. P. Ferloni, M. Mastragostino and L. Meneghello, Electrochimica Acta, 41, 27, (1996).

12. S. P. Ozkorucuklu, Y. Sahin and G. Alsancak, Sensors, 8, 8463, (2008).

13. C. Jerome, D. E. Labaye and R. Jerome, Synthetic Metals, 142, 207, (2004).

14. K.M. Cheung and D. Bloor, Polymer, 29, 1709, (1988).

15. R. P. R. Greef, L. M. Peter, D. Pletcher and J. Robinson, Instrumental Methods in Electrochemistry, Ellis Horwood Ltd., (1985).

16. M.R. Warren and J.D. Madden, Synthetic Metals, 156, 724, (2006).

17. X. Ru, W. Shi, X. Huang, X. Cui, B. Ren and D. Ge, Electrochimica Acta, 56, 9887, (2011). 